\title{
Regression of advanced maxillary sinus cancer with orbital invasion by combined chemotherapy and immunotherapy: A one-year follow-up case report
}

\author{
YU-HSUAN KUO ${ }^{1}$ and CHIH-SHIN LIN ${ }^{2,3}$ \\ Departments of ${ }^{1}$ Hematology and Oncology and ${ }^{2}$ Otolaryngology, Chi-Mei Medical Center; \\ ${ }^{3}$ Department of Speech Language Pathology, Chung Hwa University of Medical Technology, Tainan 710, Taiwan, R.O.C.
}

Received December 17, 2020; Accepted January 10, 2022

DOI: $10.3892 / \mathrm{mco} .2022 .2527$

\begin{abstract}
A case of advanced maxillary cancer undergoing a combination of chemotherapy and immunotherapy is reported. The subject a 59-year-old man had a history of nasopharyngeal carcinoma treated with concurrent chemoradiotherapy in 2004. The patient was diagnosed with a right maxillary sinus carcinoma invading the right orbital fossa (cT4aNOM0, stage IVA) in 2019. Computed tomography revealed soft tissue swelling involving the right maxillary sinus, right nasal cavity, inferior extraconal space of the right eye, right cheek and right hard palate with extension into the right pterygopalatine fossa. The patient refused surgery and radiotherapy but accepted chemotherapy in combination with immunotherapy. A marked regression of advanced maxillary sinus cancer with orbital invasion using this regimen was noted in the one-year follow-up period. A literature review on treatment for advanced maxillary sinus cancer was also conducted and the results revealed that this combination regimen may play a role in the treatment of advanced maxillary sinus cancer but further investigation is required to determine the effect of combination of chemotherapy and immunotherapy on advanced maxillary sinus cancer.
\end{abstract}

\section{Introduction}

Malignant tumors of the maxillary sinus are rare neoplasms that account for $\sim 3 \%$ of head and neck cancers and $0.5 \%$ of all malignant diseases. The annual incidence of maxillary sinus cancer is $0.5-1$ case per 100,000 of the population (1). Squamous cell carcinoma is the most common histologic type, accounting for $\sim 70-80 \%$ of maxillary sinus cancer (1).

Correspondence to: Dr Chih-Shin Lin, Department of Otolaryngology, Chi-Mei Medical Center, 901 Chung Hwa Road, Yung Kung, Tainan 710, Taiwan, R.O.C.

E-mail: ellery51.holmes@gmail.com

Key words: advanced maxillary sinus cancer, chemotherapy, immunotherapy
Most patients with maxillary sinus cancer have no symptoms in the early stage and, therefore, many of these patients are diagnosed in the advanced stage of the disease (2). At the time of diagnosis, $70-80 \%$ of maxillary sinus carcinomas present with local extension and are classified as T3 or T4 using the American Joint Committee on Cancer staging system $(3,4)$.

Maxillary sinus squamous cell carcinoma is an aggressive tumor and treatment includes surgery, radiation therapy, or a combination of both. Nevertheless, for more advanced stages, both surgery and radiotherapy have their limitations (5). Locally advanced carcinoma of the maxillary sinus has been reported to have a poor prognosis (6). Induction chemotherapy may be effective for locally advanced and technically unresectable maxillary sinus cancers resulting in successful surgery in a significant proportion of patients (6).

Immunotherapy is another possible treatment for maxillary sinus cancer. Immune checkpoint blockade has emerged as a promising treatment option. Numerous anti-PD-1 and anti-PD-L1 antibodies are under investigation, and two of them (nivolumab and pembrolizumab) were approved by the Food and Drug Administration (FDA) in 2016 for sinonasal cancers (7). PD-1 and its ligands (PD-L1 and PD-L2) are mainly involved in the modulation of T-cell activity. Tumors upregulate PD-L1 to mediate immune tolerance. Blocking the PD-1 pathway enhances T-cell-mediated tumor cell killing (8).

There have been numerous studies on the use of the combination of chemotherapy and radiotherapy for maxillary sinus cancer investigating the effect of the combination of immunotherapy and radiotherapy on maxillary sinus cancer $(8,9)$. However, to date, no study has investigated the combination of chemotherapy and immunotherapy for maxillary sinus cancer.

The case of a patient with advanced maxillary sinus cancer who refused surgery and radiotherapy is presented. After discussion, the patient elected a combination of chemotherapy and immunotherapy as initial palliative treatment. A marked regression of the tumor including orbital invasion with stable condition was noted at the follow-up of one year.

\section{Case report}

A 59-year-old man with a history of nasopharyngeal carcinoma was treated with concurrent chemoradiotherapy 


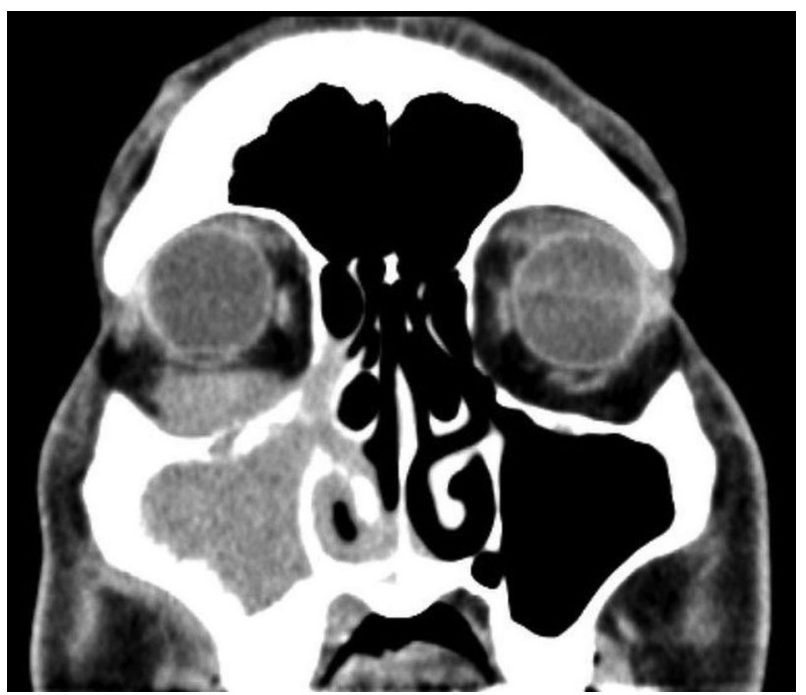

Figure 1. A suspicious soft tissue mass involving the right maxillary sinus, right nasal cavity, inferior extraconal space of the right eye, right cheek, and right hard palate.

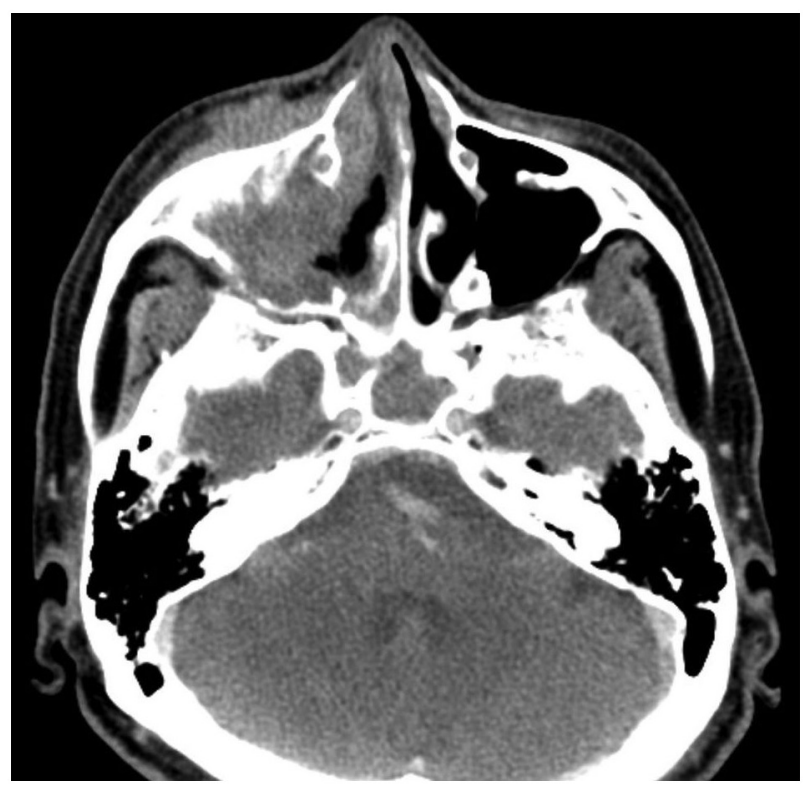

Figure 2. A suspicious soft tissue mass involving the right maxillary sinus, right nasal cavity, right cheek, and right pterygopalatine fossa extension.

in 2004. The patient experienced right maxillary swelling for several weeks with blurred vision in the right eye since February 2019. Computed tomography performed on March 29, 2019 revealed a suspicious soft tissue mass involving the right maxillary sinus, right nasal cavity, inferior extraconal space of the right eye, right cheek, and right hard palate with extension into the right pterygopalatine fossa (Figs. 1 and 2). The patient underwent biopsy through endoscopic sinus surgery at Chi-Mei Medical Center (Tainan, Taiwan). Pathology revealed infiltrative sheets of neoplastic cells featuring a syncytial growth pattern and positive for $\mathrm{p} 40$ but negative for CD56, synaptophysin, or chromogranin-A, consistent with invasive carcinoma with squamous differentiation (Fig. 3). The patient was diagnosed and staged as right

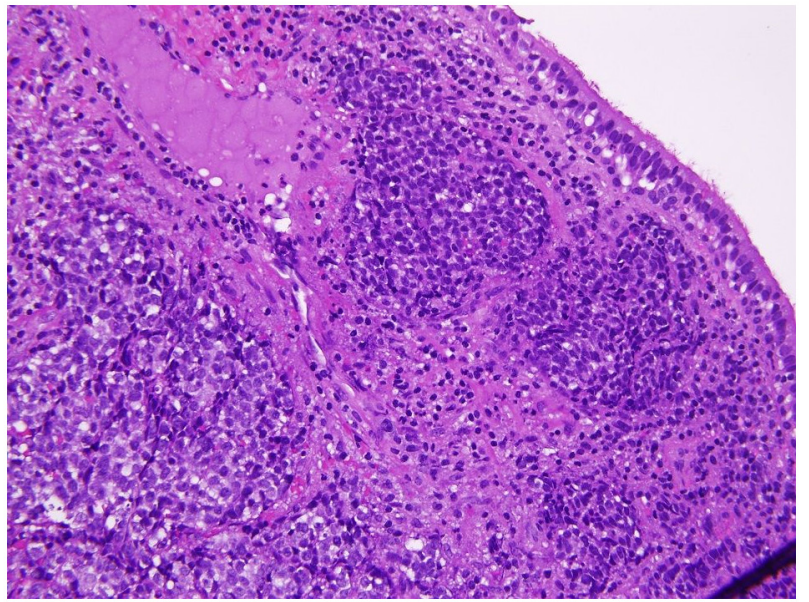

Figure 3. Pathology reveals invasive carcinoma with squamous differentiation (10x40 magnification).

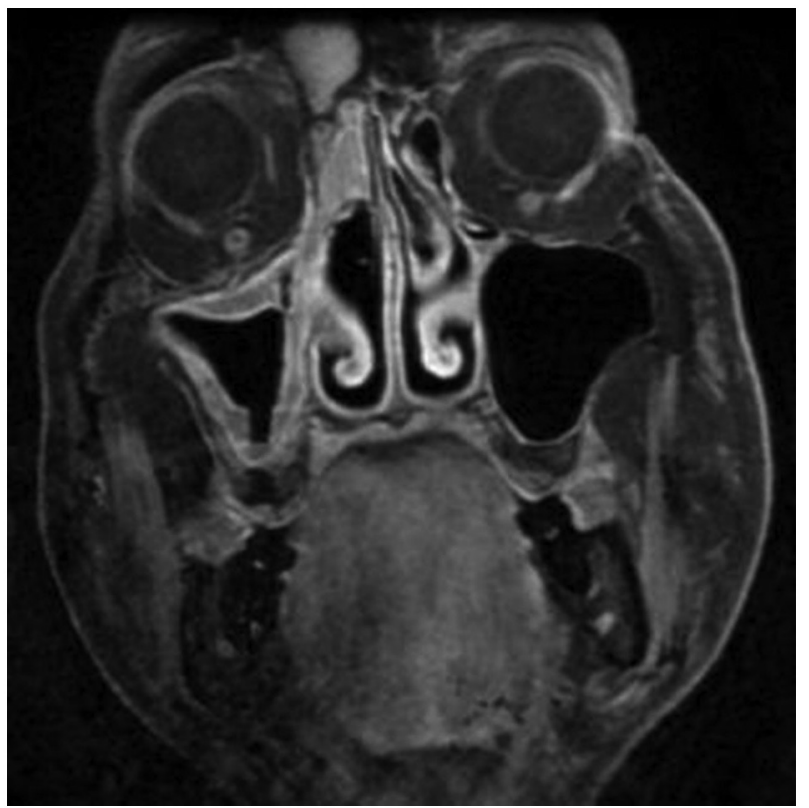

Figure 4. Marked regression of the tumor including right orbital invasion (January 6, 2020).

maxillary sinus carcinoma with invasion of the right orbital fossa, cT4aN0M0 (stage IVA).

The patient declined surgery and radiotherapy but received a combination of chemotherapy and immunotherapy as palliative treatment. Based on his height $(169 \mathrm{~cm})$ and weight $(68 \mathrm{~kg})$, the patient received a combination regimen of immunotherapy (pembrolizumab $200 \mathrm{mg}$ on day 1) and chemotherapy (cisplatin $\sim 120 \mathrm{mg}$ on day 1; fluorouracil $\sim 1,740 \mathrm{mg}$ on days 1-3) from April 15 to September 30, 2019 for eight cycles. The patient tolerated treatment well despite experiencing mild dizziness occasionally. Right maxillary swelling and right eye blurred vision gradually disappeared. Magnetic resonance imaging (MRI) performed on January 6, 2020 revealed marked regression of the tumor including right orbital invasion (Fig. 4). However, stable enhancing lesions were still present in the right pterygopalatine fossa, right foramen rotundum and right medial and lateral pterygoid plates. A residual tumor was 
Table I. Epstein-Barr virus viral load (IU/ml).

\begin{tabular}{lcccc}
\hline $2019 / 5 / 30$ & $2019 / 9 / 5$ & $2019 / 11 / 12$ & $2020 / 2 / 18$ & $2020 / 5 / 12$ \\
\hline $\begin{array}{l}\text { Not } \\
\text { detected }\end{array}$ & $\begin{array}{c}\text { Not } \\
\text { detected }\end{array}$ & $\begin{array}{c}\text { Not } \\
\text { detected }\end{array}$ & $\begin{array}{c}\text { Not } \\
\text { detected }\end{array}$ & $\begin{array}{c}\text { Not } \\
\text { detected }\end{array}$ \\
\hline
\end{tabular}

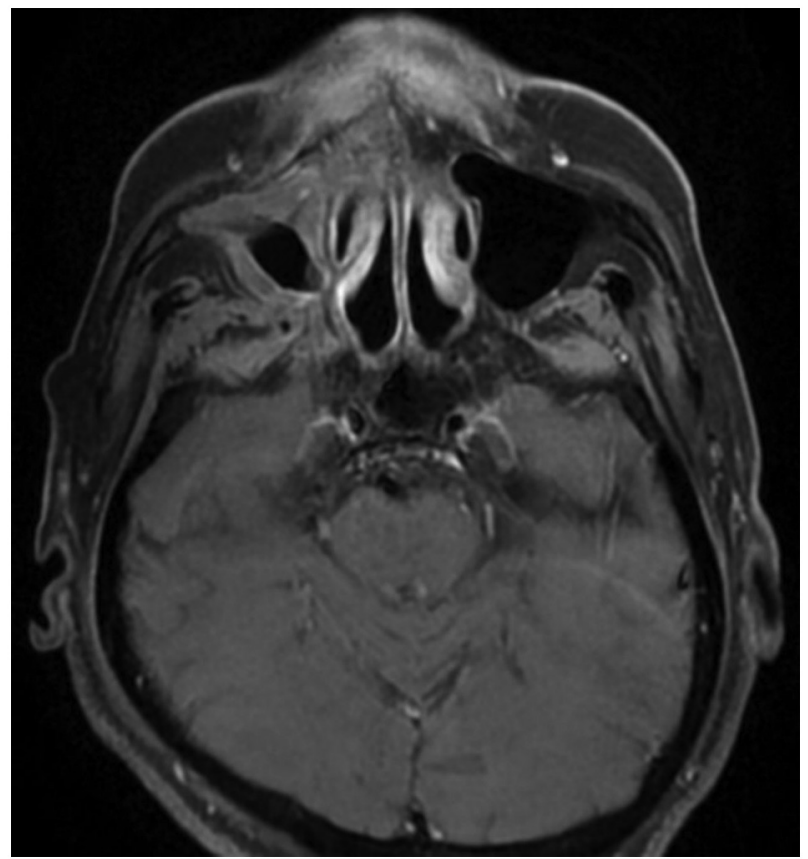

Figure 5. At the 1-year follow-up, there was still a stable enhancing lesion in the right pterygopalatine fossa, right foramen rotundum and right medial and lateral pterygoid plates. A residual tumor was suspected in the enhancing lesion.

suspected in the enhancing lesions (Fig. 5). The follow-up of Epstein-Barr virus viral load is presented in Table I. On the 1-year follow-up visit, the patient was in stable condition. MRIs were planned for this patient in future follow-ups.

\section{Discussion}

The main treatment options for maxillary sinus cancer include surgery, radiation therapy, and a combination of both. Since the patient in our case refused surgery and radiotherapy, other possibilities were discussed with him. The patient decided to undergo the combination of chemotherapy and immunotherapy as palliative treatment. There have been limited studies in the literature on the combination of chemotherapy and immunotherapy for advanced maxillary sinus. Thus, it is valuable to investigate its effects.

Concurrent chemoradiation was not used due to the patient's history of nasopharyngeal cancer treated with radiotherapy. Radiation toxicity was considered. According to the evaluation of the radiation oncologist, blindness of the right eye from cancer radiotherapy could occur.

Numerous anti-PD-1 and anti-PD-L1 antibodies are under investigation, and two of them (nivolumab and pembrolizumab) were approved by the FDA in 2016 for sinonasal cancers (8).
Blocking the PD-1 pathway enhances T cell-mediated killing of tumor cells (8). The results of the immunohistochemical analysis of the tumor of the patient revealed that it was positive for PD-L1 and the tumor proportion score was $10 \%$. It is known that high PD-L1 expression levels and a high mutational burden are correlated with higher response rates to PD-1-based immunotherapies (8). Previous studies have revealed the effect of combining immunotherapy and radiotherapy on maxillary sinus cancer $(8,9)$ indicating favorable responses. Thus, it may be used as an alternative for this patient if he elected radiotherapy, despite that radiation toxicity must be considered due to the history of the patient with radiotherapy for nasopharyngeal cancer.

There are other examples of combination of chemotherapy and immunotherapy for other tumors. One example is gemcitabine and cisplatin plus pembrolizumab as neoadjuvant therapy before radical cystectomy in patients with muscle-invasive bladder cancer (10). Another example is pembrolizumab plus chemotherapy in metastatic non-small cell lung cancer (11). The combination of chemotherapy and immunotherapy can achieve additive or synergistic clinical activity. Two major means through which chemotherapy promotes tumor immunity are by inducing immunogenic cell death as part of its intended therapeutic effect and by disrupting strategies that tumors use to evade immune recognition (12).

It was noted that induction chemotherapy for locally advanced maxillary sinus cancer increased the possibility of complete resection with orbital preservation as well as tumor downstaging. The MRI of the patient on January 6, 2020 still showed a stable enhancing lesion in the right pterygopalatine fossa, right foramen rotundum, and right medial and lateral pterygoid plates. A residual tumor was suspected and considered technically unresectable due to the involvement of the pterygoid plates. However, regression of advanced maxillary sinus cancer including right orbital invasion was observed. The effect of the combination of chemotherapy and immunotherapy may play a role in downstaging tumors, although further investigation is required.

In conclusion, marked regression of advanced maxillary sinus cancer with orbital invasion through the combination of chemotherapy and immunotherapy was observed in this one-year follow-up case. Further investigation is still needed on the effect of the combination of chemotherapy and immunotherapy on advanced maxillary sinus cancer.

\section{Acknowledgements}

Not applicable.

\section{Funding}

No funding was received.

\section{Availability of data and materials}

The datasets used during the present study are available from the corresponding author on reasonable request.

\section{Authors' contributions}

CSL performed the surgical procedures. CSL and YHK conceived the study, wrote the manuscript and confirm the 
authenticity of all the raw data. Both authors read and approved the manuscript and agree to be accountable for all aspects of the research in ensuring that the accuracy or integrity of any part of the work are appropriately investigated and resolved.

\section{Ethics approval and consent to participate}

Not applicable.

\section{Patient consent for publication}

Written informed consent was obtained from the patient for publication of this case report.

\section{Competing interests}

The authors declare that they have no competing interests.

\section{References}

1. Won HS, Chun SH, Kim BS, Chung SR, Yoo IeR, Jung CK Kim YS, Sun DI, Kim MS and Kang JH: Treatment outcome of maxillary sinus cancer. Rare Tumours 1: e36, 2009.

2. Praveena NM and Maragathavalli G: Carcinoma of the maxillary antrum: A case report. Cureus 10: e2614, 2018

3. Dulguerov P, Jacobsen MS, Allal AS, Lehmann W and Calcaterra T: Nasal and paranasal sinus carcinoma: Are we making progress? A series of 220 patients and a systematic review. Cancer 92: 3012-3029, 2001
4. Dooley L and Shah J: Management of the neck in maxillary sinus carcinomas. Curr Opin Otolaryngol Head Neck Surg 23: 107-114, 2015.

5. Ashraf M, Biswas J,Dam A, Bhowmick A, Jha, Sing V and Nayak S: Results of treatment of squamous cell carcinoma of maxillary sinus: A 26-year experience. World J Oncol 1: 28-34, 2010.

6. Noronha V, Patil VM, Joshi A, Krishna MV, Dhumal S, Juvekar S, Pai P, Chatturvedi P, Chaukar DA, Agarwal JP, et al: Induction chemotherapy in technically unresectable locally advanced carcinoma of maxillary sinus. Chemother Res Pract 2014: 487872, 2014.

7. Xiang JJ, Uy NF, Minja FJ, Verter EE and Burtness BA: Hyperprogression after one dose of nivolumab in sinonasal cancer: A case report. Laryngoscope 130: 907-910, 2020.

8. Yazici G, Gullu I, Cengiz M, Elmali A, Yilmaz MT, Aksoy S, Yuce Sari S and Ozyigit G: The synergistic effect of immune checkpoint blockade and radiotherapy in recurrent/metastatic sinonasal cancer. Cureus 10: e3519, 2018.

9. Guram K, Nunez M, Einck J, Mell LK, Cohen E, Sanders PD, Miyauchi S, Weihe E, Kurzrock R, Boles S and Sharabi AB: Radiation therapy combined with checkpoint blockade immunotherapy for metastatic undifferentiated pleomorphic sarcoma of the maxillary sinus with a complete response. Front Oncol 8: 435, 2018.

10. Rose TL, Harrison MR, Deal AM, Ramalingam S, Whang YE, Brower B, Dunn M, Osterman CK, Heiling HM, Bjurlin MA, et al: Phase II study of gemcitabine and split-dose cisplatin plus pembrolizumab as neoadjuvant therapy before radical cystectomy in patients with muscle-invasive bladder cancer. J Clin Oncol 39: 3140-3148, 2021.

11. Gandhi L, Rodríguez-Abreu D, Gadgeel S, Esteban E, Felip E, De Angelis F, Domine M, Clingan P, Hochmair MJ, Powell SF, et al: Pembrolizumab plus chemotherapy in metastatic non-small-cell lung cancer. N Engl J Med 378: 2078-2092, 2018.

12. Emens LA and Middleton G: The interplay of immunotherapy and chemotherapy: Harnessing potential synergies. Cancer Immunol Res 3: 436-443, 2015. 